\title{
ARTí́CULO
}

\section{Ontogenia de la concha larval y postlarval de cuatro especies de bivalvos de la costa del Pacífico sureste}

\author{
Larval and postlarval shell ontogeny of four bivalve species \\ from the Southeast Pacific coast
}

\section{Paola Díaz ${ }^{1}$ y Bernardita Campos ${ }^{1}$}

\begin{abstract}
'Facultad de Ciencias del Mar y de Recursos Naturales, Universidad de Valparaíso, Casilla 5080, Reñaca, Viña del Mar, Chile. bernardita.campos@uv.cl

Abstract.- Larval and early postlarval shell morphology and morphometry of the bivalves Venus antiqua, Mulinia edulis, Ensis macha and Hiatella solida were studied. The specimens examined were obtained from plankton samples and/or from experimental laboratory cultures. The size ranges of the specimens studied were: $123-370 \mu \mathrm{m}$ in V. antiqua, $140-580 \mu \mathrm{m}$ in M. edulis, 157-670 $\mu \mathrm{m}$ in E. macha and 145-1270 $\mu \mathrm{m}$ in H. solida. The shell features analyzed were: general shape, umbo, shoulders, provinculum, and hinge lateral system. The total shell length and height, length of shoulders, provinculum and prodissoconch I and II lengths were measured. V. antiqua and M. edulis shells have similar appearance and provinculum; they differ only in the shape of the shoulders and in the lateral hinge system. E. macha and $\mathrm{H}$. solida are clearly distinguishable from the other species studied. Each species showed characteristics of the genus and family to which they belong; only $M$. edulis differed from other species of the same genus in its provincular structure, umbo shape and size at which the ligament develops. All species exhibited allometric growth, with significant different growth rates $(P<0.05)$ in both developmental stages. The traditional identification method based on shell morphology is still a valid and indispensable tool for the recognition of early life stages of bivalves collected in plankton and benthos although complementary identification techniques based on DNA analysis are strongly recommended.
\end{abstract}

Key words: Bivalvia, identification, hinge development, Venus antiqua, Mulinia edulis, Ensis macha, Hiatella solida

\begin{abstract}
Resumen.- Este estudio caracterizó la morfología y morfometría de la concha de larvas y postlarvas de los bivalvos Venus antiqua, Mulinia edulis, Ensis macha y Hiatella solida. Los ejemplares se obtuvieron del plancton y/o de cultivos en laboratorio. Los rangos de talla estudiados fueron: 123-370 $\mu \mathrm{m}$ en V. antiqua, 140-580 $\mu \mathrm{m}$ en M. edulis, 157-670 $\mu \mathrm{m}$ en $\mathrm{E}$. macha y 145-1270 $\mu \mathrm{m}$ en $\mathrm{H}$. solida. Los caracteres analizados en la concha fueron: forma general, umbo, hombros, provínculo y sistema lateral de charnela. Se midió largo y alto total de la concha, largo de hombros, del provínculo y de las prodisoconchas I y II. Las conchas de V. antiqua y $\mathrm{M}$. edulis presentan similar apariencia externa y provincular y se diferencian solo en forma de hombros y sistema lateral de charnela. E. macha y $\mathrm{H}$. solida son claramente diferentes de las otras especies estudiadas. Cada especie presentó características propias del género y familia a la que pertenece, excepto M. edulis que se diferenció de otras especies del mismo género en su estructura provincular, forma del umbo y talla de aparición del ligamento. Las 4 especies presentaron crecimiento alométrico y fueron significativamente diferentes en la forma de la concha y en las tasas de cambio de talla $(P<0,05)$. El método de identificación basada en la morfología de la concha se mantiene como una herramienta vigente e indispensable para el reconocimiento de los primeros estados de vida de bivalvos recolectados del plancton y del bentos especialmente en monitoreos sistemáticos aunque se recomienda complementarla con otras técnicas como el análisis de ADN.
\end{abstract}

Palabras clave: Bivalvia, identificación, desarrollo charnelar, Venus antiqua, Mulinia edulis, Ensis macha, Hiatella solida

\section{INTRODUCCIÓN}

La mayoría de los moluscos bivalvos, como resultado de la fertilización, produce secuencialmente los estados pelágicos de embrión, larva trocófora y larva velígera, para transformarse en postlarvas bentónicas, después de un proceso de metamorfosis (Zardus \& Martel 2002). Al igual que en otros invertebrados marinos, las larvas y postlarvas son importantes ya que aportan información acerca de la diversidad de especies en un área geográfica, del estado de los bancos naturales, de las épocas de reproducción, de asentamiento y del reclutamiento (Shanks 2001). 
La concha de los bivalvos se origina durante la embriogenesis, con el funcionamiento de una glándula de la concha, en el estado de larva trocófora (Moueza et al. 2006). La concha inicial secretada por esta glándula se denomina prodisoconcha I y se caracteriza por presentar una superficie homogénea y tener forma de $\mathrm{D}$, dando el nombre de 'larva D' a la larva velígera temprana. La secreción de concha que el manto de la larva produce en el margen de la prodisoconcha I origina la prodisoconcha II, que se caracteriza por presentar líneas concéntricas. Durante la metamorfosis se produce una discontinuidad en el crecimiento de la concha quedando una marca evidente que diferencia la concha larval de aquella formada por la postlarva y denominada disoconcha (Jablonski \& Lutz 1980).

La concha se utiliza para la identificación morfológica de larvas y postlarvas de bivalvos (por ejemplo, Rees 1950, Savage \& Golberg 1976, Le Pennec 1980, Lutz et al. 1982, Tremblay et al. 1987, Goodsell et al. 1992, Slater 2005, Schejter et al. 2010), debido a que presenta en sus estados más avanzados, una morfología que la diferencia de las conchas de otras especies y persiste inalterada por largo tiempo aún después de la muerte del animal, a diferencia de lo que ocurre con el cuerpo blando, que puede ser modificado por factores ambientales y es degradado y/o se modifica con los líquidos fijadores utilizados para preservar estos estadios.

Los criterios morfológicos utilizados en la caracterización de la concha de larvas y postlarvas de bivalvos incluyen su forma general, forma del umbo y de los hombros, color, microestructura de las prodisoconchas I y II, y de la disoconcha, además de las características de la charnela (Rees 1950, Brink 2001). El análisis morfológico frecuentemente se complementa con la descripción de algunas características morfométricas (largo y alto de las prodisoconchas I y II y de los hombros) (Malchus 2006) ${ }^{1}$ y con las relaciones entre el largo, el ancho y el alto de la concha (De Schweinitz \& Lutz 1976, Lutz \& Jablonski 1978, Hendriks et al. 2005, Paugam et al. 2006, Schejter et al. 2010). Este conjunto de caracteres morfológicos y morfométricos es el que permite la identificación precisa de larvas y postlarvas de una especie determinada, aunque se debe tener presente que se trata de un método subjetivo y que estos estados del desarrollo pueden presentar una considerable plasticidad fenotípica, tal como lo señalan Bownes et al. (2008).
A pesar del desarrollo de diversas técnicas que permiten acelerar el proceso de identificación a nivel específico (análisis de imágenes, inmunológicos, electroforéticos y de análisis de secuencias del ADN, entre otros) (e.g., Goffredi et al. 2006, McManus \& Katz 2009, Pardo et al. 2009), el análisis morfológico se mantiene como método estándar de identificación en los análisis sistemáticos de muestras de plancton y bentos, ya que este puede ser llevado a cabo en cualquier laboratorio de investigación que disponga al menos de un microscopio estereoscópico con luz transmitida. Sin embargo, hay que considerar que estos análisis morfológicos son muy laboriosos y de alto costo si se trata de procesar un gran número de muestras.

Aunque para la costa de Chile se han señalado más de 230 especies de bivalvos (Valdovinos 1999), los estudios acerca de la morfología de larvas y postlarvas con fines de identificación son escasos y se concentran sólo en algunas especies de las familias Mytilidae, Ostreidae, Pectinidae, Pholadidae y Teredinidae (Chanley \& Dinamani 1980, Campos \& Ramorino 1981, 1990; Ramorino \& Campos 1983, Bellolio et al. 1993, 1994). Como a pesar de los esfuerzos, persiste la incapacidad de identificar las larvas planctónicas y los primeros estados bentónicos de la mayoría de las especies de bivalvos que habitan las costas del Pacífico sureste, el objetivo de este trabajo fue describir los cambios ontogenéticos que ocurren en las conchas de los bivalvos Venus antiqua (King, 1831), Mulinia edulis (King, 1831), Ensis macha (Molina, 1782) y Hiatella solida (Sowerby, 1834), para incrementar el número de especies que puedan ser identificadas a nivel de larvas y postlarvas, de modo de permitir el desarrollo de estudios ecológicos especie-específicos relacionados con estos estados, considerando que las 3 primeras especies constituyen recursos económicos en Chile.

\section{MATERIALES Y MÉTODOS}

Las larvas y postlarvas de Venus antiqua, Mulinia edulis y Ensis macha se obtuvieron de cultivos experimentales en laboratorio y las de Hiatella solida se recolectaron en muestreos planctónicos y bentónicos realizados frente a Valparaíso (3257,4’S, $\left.71^{\circ} 33,4^{\prime} \mathrm{W}\right)$, entre julio 1982 y enero 1984, y en Putemún $\left(41^{\circ} 25,5^{\prime} \mathrm{S}, 73^{\circ} 44,5^{\prime} \mathrm{W}\right)$, Chile, en noviembre 1981, marzo 1982, febrero y noviembre 1983.

\footnotetext{
${ }^{1}$ Malchus N. 2006. Easy to see, difficult to describe: towards a standardized description of bivalve Iarval shell shape. In: Malchus N \& JM Pons (eds). International Congress on Bivalvia: Organisms, Diversity \& Evolution, Electronic Supplement 2006-16, part 1: p. 53. <http://www.senckenberg.de/odes/06-16/Malchus_Prodissoconch-DescriptionTool.pdf>
} 
Todos los ejemplares estudiados fueron fijados en etanol $90 \%$ con glicerofosfato de sodio para evitar la descalcificación de las valvas (Turner 1976). Los ejemplares preservados fueron sumergidos en una solución de hipoclorito de sodio al $3 \%$ por 5 min para digerir el tejido blando y desarticular las valvas, las cuales una vez abiertas, fueron enjuagadas con agua destilada y mantenidas en etanol para su posterior observación. Las valvas separadas de cada ejemplar se montaron sobre un portaobjetos, en una gota de glicerol. Las observaciones y mediciones se realizaron con un microscopio Leitz ${ }^{\circledR}$ DMLB, provisto de ocular reticulado y el registro fotográfico se hizo con una cámara Leica ${ }^{\circledR}$ MPS 60, acoplada al microscopio.

Las características morfológicas consideradas en la descripción fueron: forma de la concha y del umbo, presencia de estrías en la superficie externa de las valvas, color y grado de transparencia de la concha, estructura charnelar y del sistema lateral de la charnela, presencia, disposición y forma de la hendidura del ligamento. La terminología utilizada corresponde a Rees (1950), Chanley \& Andrews (1971), Ramorino \& Campos (1983) y Brink (2001). Se excluyó del estudio el estado de larva 'D' debido a su similitud en las diferentes especies y a la carencia de caracteres morfológicos que contribuyeran a su identificación.

La caracterización morfométrica se basó en la medición de: largo total (LT), alto total (AT), largo de la prodisoconcha I (LPI), largo del hombro anterior (LHa), largo del hombro posterior (LHp), largo provincular (LPv) y largo de prodisoconcha II (LPII) (Fig. 1), según la terminología de Ramorino \& Campos (1983).

Para cada especie se establecieron las siguientes correlaciones lineales propuestas por Lutz (1985): alto total / largo total, largo total / largo provincular, alto total / largo provincular, largo del hombro posterior / largo del hombro anterior.

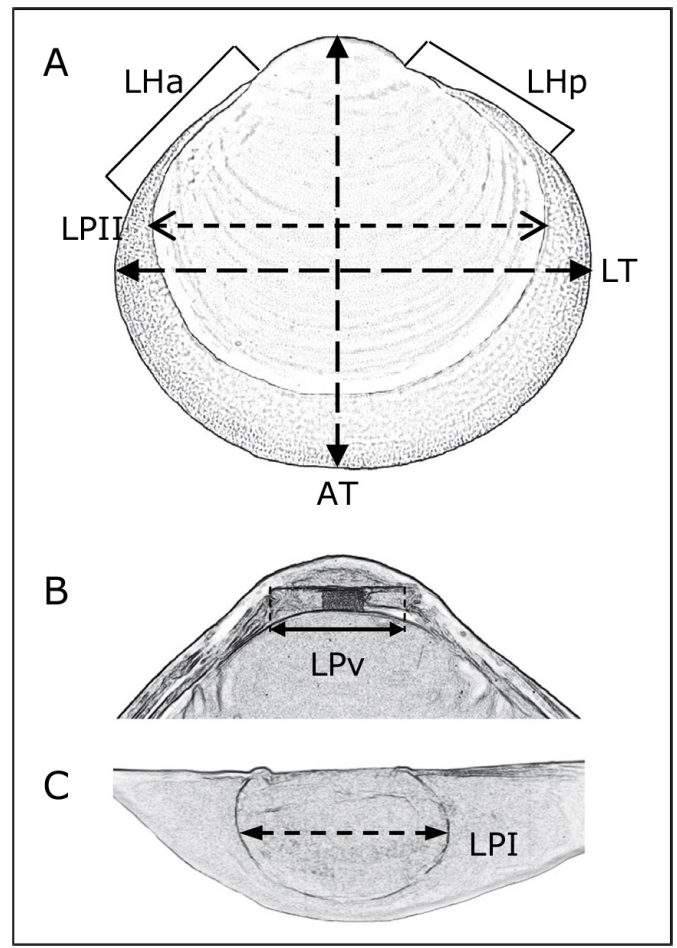

Figura 1. Terminología utilizada para describir las dimensiones de la concha. A: vista lateral externa de la concha; B: vista lateral interna; C: vista dorsal. LT: largo total. AT: alto total. LHa: longitud del hombro anterior. LHp: longitud del hombro posterior. LPv: longitud provincular. LPI: longitud de prodisoconcha I. LPII: longitud de prodisoconcha II / Terminology used to describe shell dimensions. A: external lateral view of the shell; B: internal lateral view; C: dorsal view. LT: total length. AT: total height. LHa: anterior shoulder length. LHp: posterior shoulder length. LPv: provincular length. LPI: prodissoconch I length. LPII: prodissoconch II length 
En $V$. antiqua la morfometría se analizó en 127 larvas en estado umbonado (123-300 $\mu \mathrm{m}$ LT) y 9 postlarvas (325$370 \mu \mathrm{m}$ LT); en M. edulis, en 114 larvas (140-355 $\mu \mathrm{m} \mathrm{LT)} \mathrm{y}$ 42 postlarvas (300-580 $\mu \mathrm{m} \mathrm{LT}$ ); en E. macha, en 45 larvas (157-325 $\mu \mathrm{m}$ LT) y 62 postlarvas $(280-670 \mu \mathrm{m} \mathrm{LT})$ y en $H$. solida, en 144 larvas $(145-370 \mu \mathrm{m}$ LT) y 48 postlarvas (440-1270 $\mu \mathrm{m} \mathrm{LT)}$.

Las relaciones morfométricas se describieron en base a ecuaciones de regresión lineal (Zar 1984). La determinación de diferencias significativas en la relación AT-LT y en las tasas de crecimiento se realizó mediante un análisis de covarianza (ANCOVA) (Zar 1984); en ambos casos se utilizó el programa PAST versión 2.16 (Hammer et al. 2001). En el análisis estadístico no se incluyó a $V$. antiqua en estado postlarval, por contar con menos de 10 individuos.

\section{Resultados}

\section{MoRFología}

\section{VENUS ANTIQUA}

La concha larval es equivalva y presenta un umbo bajo ampliamente curvado que se hace más prominente al final de este estado, formando una línea continua con el resto del margen valvar, el cual es casi equilateral; el borde anterior de la concha es más largo y agudo que el posterior y el margen ventral es semicircular. Los hombros son cortos, redondeados y bajos; el anterior es más largo que el posterior (Fig. 2A). La prodisoconcha I presenta una superficie homogénea y la prodisoconcha II, tenues líneas concéntricas. La línea paleal y las impresiones de los músculos aductores no son visibles con microscopía óptica. Las valvas son incoloras y transparentes. Internamente, el provínculo (charnela) es delgado, con proyecciones denticulares muy pequeñas en la parte central y anterior de las valvas derecha e izquierda respectivamente (Fig. 2B y D). El ligamento tiene forma elipsoide y presenta una hendidura posterior (Fig. 2B y D). El sistema lateral de charnela comienza a desarrollarse cuando la larva alcanza cerca de $245 \mu \mathrm{m}$ LT, consta de 2 rebordes y 2 canaletas, ubicados en la valva izquierda y derecha, respectivamente (Fig. 2B y D). Alrededor de los $280 \mu \mathrm{m}$ LT comienza el desarrollo de un diente lateral anterior en la valva derecha de la concha larval (Fig. 2B y D).

Las postlarvas presentan una concha inequilateral; el borde anterior es más largo y agudo que el posterior. El margen ventral es semicircular y el umbo tiende a ser prominente. Los hombros son cortos, rectos y bajos, con el anterior más largo que el posterior (Fig. 2A). La disoconcha es incolora, opaca y presenta una textura granular; las líneas concéntricas de crecimiento no son distinguibles con microscopía óptica, sino hasta cuando la postlarva mide cerca de $360 \mu \mathrm{m}$ LT. La línea paleal y las impresiones de los músculos aductores aún no son visibles (Fig. 2A). El provínculo es más grueso que en larvas. En ejemplares cercanos a $360 \mu \mathrm{m}$ LT, los dientes centrales de la valva derecha conforman un solo diente, los restantes no evolucionan y dejan de ser observables en ejemplares cercanos a $370 \mu \mathrm{m} \mathrm{LT}$, (Fig. 2C y E). La hendidura del ligamento presenta una forma circular (Fig. 2C y E). El sistema lateral de la charnela está compuesto de 2 dientes anteriores, uno sólido y otro lamelar, ubicados en la valva derecha e izquierda, respectivamente (Fig. 2C y E).

\section{MULINIA EDULIS}

Las larvas analizadas se encontraban en estado umbonado. La concha larval es equivalva e inequilateral, con el borde anterior más largo y agudo que el posterior. El margen ventral es semicircular. El umbo es redondeado y amplio, aunque al término del período larval tiende a ser angular. Los hombros son cortos, rectos y bajos, generalmente con el anterior más largo que el posterior (Fig. 3A). Las valvas de la concha larval son incoloras y transparentes. La prodisoconcha I presenta una textura granular uniforme, mientras que la prodisoconcha II exhibe líneas concéntricas. La línea paleal no es observable; las impresiones de los músculos aductores son visibles a partir de los $290 \mu \mathrm{m}$ LT (Fig. 3A). Internamente, el provínculo es delgado y solo desarrolla pequeños dientes en la parte centro-posterior de la valva derecha (Fig. 3B y D). La hendidura del ligamento es observable a partir de los 170 $\mu \mathrm{m}$ LT, como una zona oscura hacia la parte posterior del provínculo, aunque más visible alrededor de los $300 \mu \mathrm{m}$ LT. En vista dorsal, la hendidura se observa algo elipsoide (Fig. 3B y D). El sistema lateral de la charnela comienza a desarrollarse cuando los individuos alcanzan alrededor de $300 \mu \mathrm{m}$ LT; está compuesto de un reborde posterior y una canaleta anterior en la valva derecha, además de un diente especial anterior en la valva derecha (Fig. 3B y D).

Las postlarvas presentan el margen valvar continuo e inequilateral, con el borde anterior más largo y agudo que el posterior y con el margen ventral semicircular. El umbo se observa prominente. Los hombros son cortos, redondeados y altos (Fig. 3A). La disoconcha presenta líneas concéntricas. La línea paleal y las impresiones de los músculos aductores no son visibles (Fig. 3A). Las 


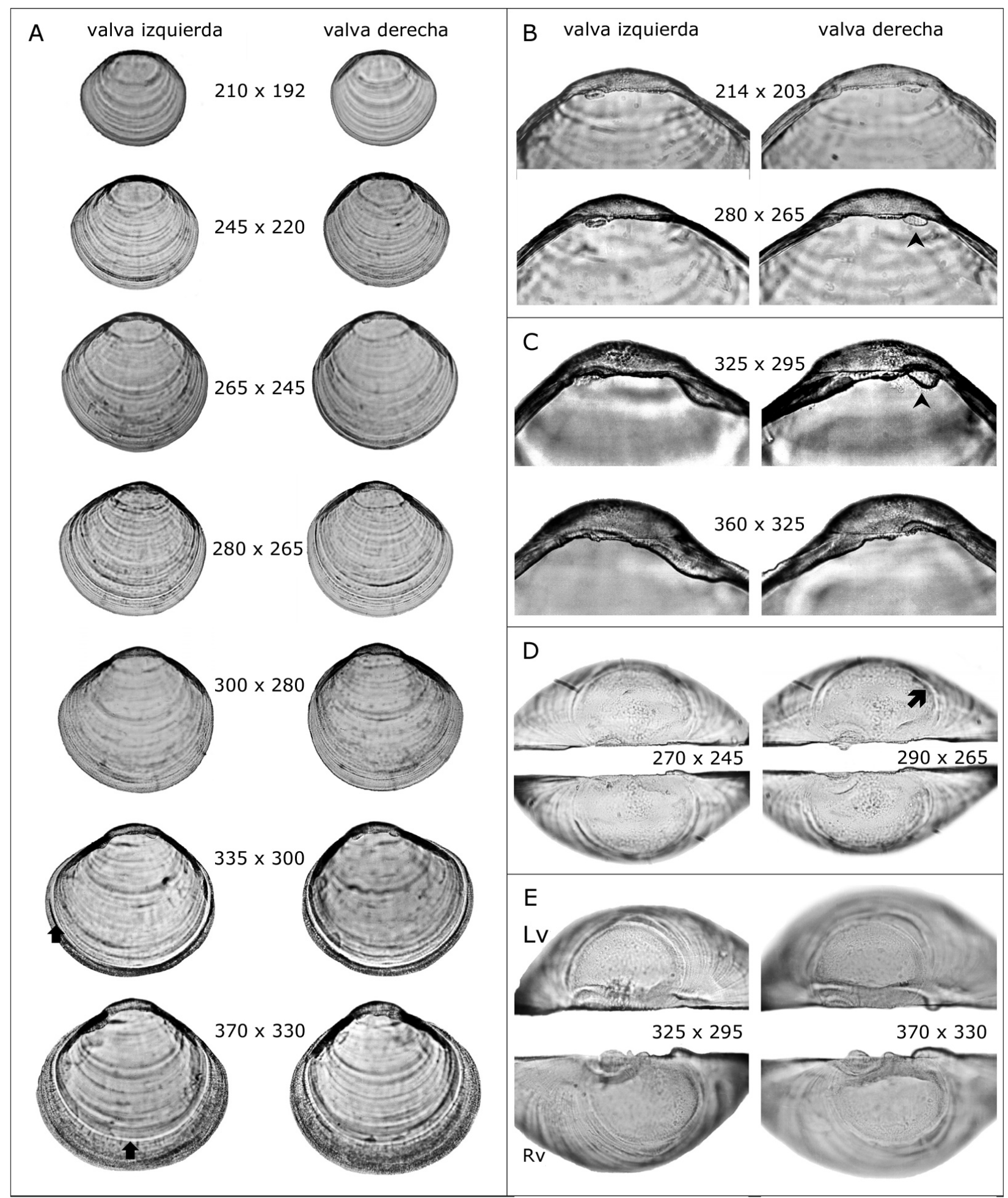

Figura 2. Venus antiqua. A. Vista lateral externa de la concha. B y C. Vista lateral interna de la concha larval y postlarval, respectivamente. D y E. Vista dorsal de la concha larval y postlarval, respectivamente. Vd: valva derecha. Vi: valva izquierda. : línea metamórfica. $A$ : hendidura del ligamento. $\boldsymbol{\lambda}$ : límite prodisoconcha I - prodisoconcha II. Dimensiones (LT x AT) en $\mu \mathbf{m}$ / Venus antiqua. A. External lateral view of the shell. B and C. Internal lateral view of the larval and postlarval shell, respectively. D and E. dorsal view of the larval and postlarval shell, respectively. Vd: right valve. Vi: left valve. A : metamorphic line. A : ligament groove. $\boldsymbol{\lambda}$ : prodissoconch I-prodissoconch II limit. Dimensions (LT x AT) in $\mu \mathrm{m}$ 


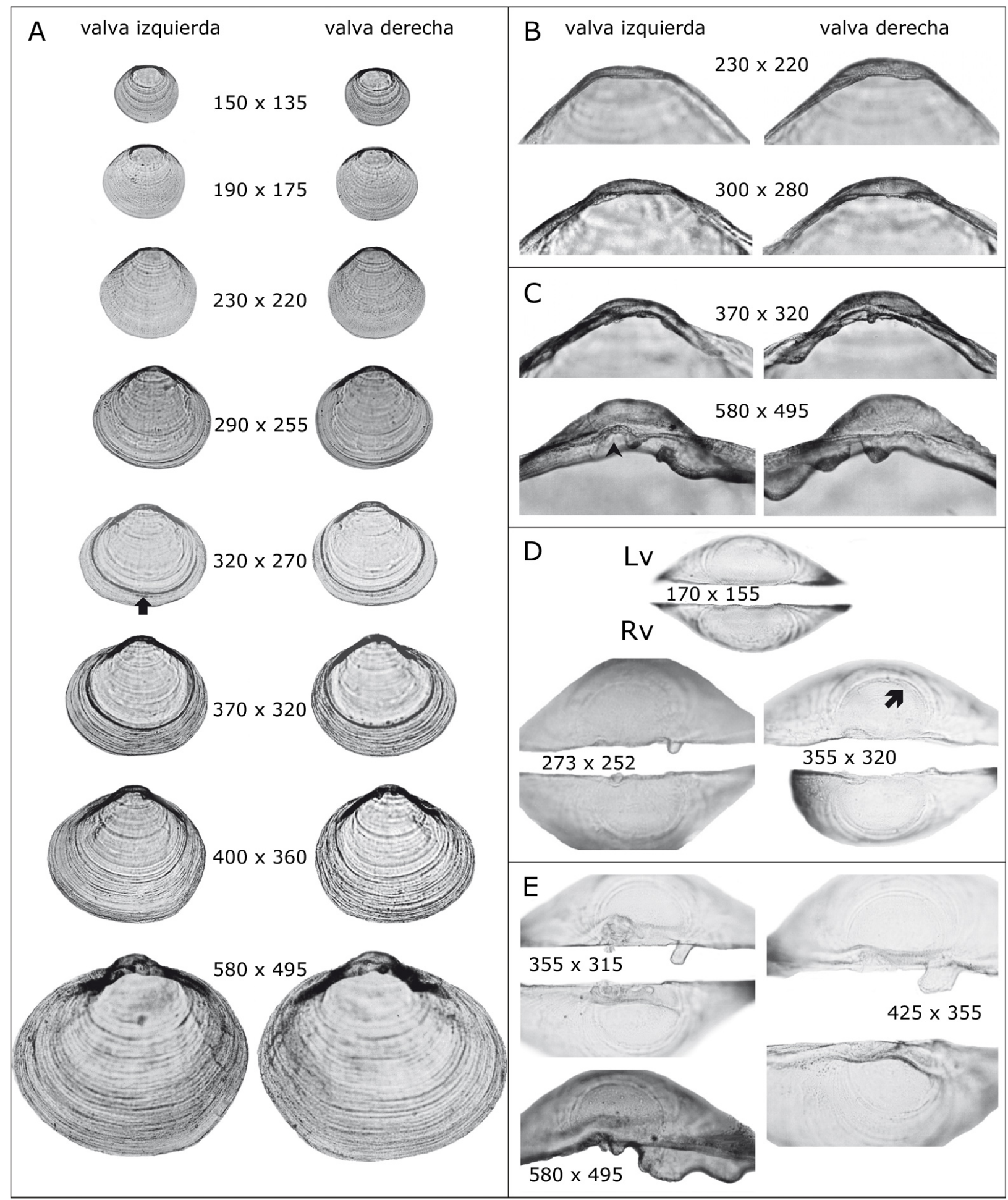

Figura 3. M ulinia edulis. A. Vista lateral externa de la concha. B y C. Vista lateral interna de la concha larval y postlarval, respectivamente. D y E. Vista dorsal de la concha larval y postlarval, respectivamente. Vd: valva derecha. Vi: valva izquierda. $\mathbf{A}$ : línea metamórfica. $\boldsymbol{A}$ : hendidura del ligamento. ה: límite prodisoconcha I - prodisoconcha II. Dimensiones (LT x AT) en $\mu \mathrm{m}$ / Mulinia edulis. A External lateral view of the shell. B and C. Internal lateral view of the larval and postlarval shell, respectively. D and E. Dorsal view of the larval and postlarval shell, respectively. Vd: right valve. Vi: left valve. $\mathbf{A}$ : metamorphic line. $\boldsymbol{A}$ : ligament groove. $\boldsymbol{\pi}$ : prodissoconch I-prodissoconch II limit. Dimensions (LT x AT) in $\mu \mathrm{m}$ 
valvas son incoloras y opacas. El provínculo en las postlarvas es más grueso que en las larvas. Alrededor de los $350 \mu \mathrm{m}$ LT se desarrolla un diente central en la valva derecha y a los $580 \mu \mathrm{m}$ LT se desarrolla un diente provincular central en la valva izquierda (Fig. 3C y E). La forma de la hendidura que aloja al ligamento fue muy variable entre los ejemplares examinados (Fig. 3C y E). El sistema lateral de charnela continúa evolucionando hacia la parte anterior; el diente anterior izquierdo crece hasta ocupar casi la totalidad del hombro, alrededor de los 580 $\mu \mathrm{m}$ LT, mientras que en la valva derecha se desarrollan 2 dientes laterales (Fig. 3C y E).

\section{ENSIS MACHA}

Las larvas analizadas se encontraban en estado umbonado. La concha presenta un margen valvar continuo, alargado e inequilateral. El borde anterior es más largo y agudo que el posterior y el margen ventral es semicircular. El umbo difiere en ambas valvas, siendo más alto en la valva derecha. Los hombros son redondeados y relativamente altos (Fig. 4A). Las valvas son incoloras y transparentes. La prodisoconcha I es homogénea mientras que la prodisoconcha II presenta tenues líneas concéntricas. La línea paleal e impresiones de los músculos aductores son visibles ya cerca de los $260 \mu \mathrm{m}$ LT (Fig. 4A). El provínculo es delgado, con 2 dientes ubicados en cada extremo en la valva derecha y un diente rectangular central en la valva izquierda (Fig. 4B y D). El ligamento, de ubicación posterior, fue observable en la valva izquierda, a partir de los $320 \mu \mathrm{m}$ LT. El sistema lateral de la charnela está formado por 2 rebordes, uno a cada lado de la valva izquierda, y 2 canaletas ubicadas una a cada lado de la valva derecha (Fig. 4B y D).

En postlarvas, el margen valvar se alarga hacia la parte anterior haciendo la concha inequilateral. El borde ventral presenta una forma elipsoide, inclinada hacia la parte posterior (Fig. 4A). Alrededor de los $400 \mu \mathrm{m}$ LT, el umbo se desarrolla de una forma ampliamente redondeada a prominente, sin embargo se mantiene más alto en la valva derecha que en la izquierda (Fig. 4A). Los hombros mantienen su forma relativamente recta, aunque a partir de los $400 \mu \mathrm{m}$ LT, el hombro posterior cambia y se observa más redondeado que el anterior (Fig. 4A). Las valvas son incoloras y opacas. La textura de la disoconcha es muy similar a la de la prodisoconcha II, con tenues líneas concéntricas y el límite entre ellas es poco distinguible (Fig. 4A). El provínculo postlarval es más grueso que en larvas y sólo se diferencia cuando la concha alcanza los $400 \mu \mathrm{m}$ LT, con el desarrollo de un diente anterior en la valva derecha. Cuando la concha alcanza los $475 \mu \mathrm{m}$ LT, este diente se observa agudo y alargado hacia la parte anterior, mientras que en la valva izquierda se desarrolla un diente rectangular central. Alrededor de los $600 \mu \mathrm{m}$ LT, la valva derecha exhibe un diente adicional posterior, mientras que el diente anterior desarrolla una base rectangular. En la valva izquierda el diente rectangular central desarrolla una protuberancia hacia la parte anterior; a los $670 \mu \mathrm{m}$ LT este último diente se divide en 2 (Fig. 4C y E). Entre los 400 y $440 \mu \mathrm{m}$ LT, las postlarvas desarrollan una hendidura posterior al provínculo sobre la cual es posible observar vestigios del ligamento (Fig. 4C y E). El sistema lateral de la charnela, en ejemplares de $670 \mu \mathrm{m}$ LT, presenta en la valva izquierda, un diente lateral anterior y otro posterior, con mayor desarrollo del primero (Fig. 4C y E).

\section{HIATELLA SOLIDA}

Todas las larvas de $H$. solida examinadas en el presente trabajo se encontraban en estado umbonado. Las larvas presentan tempranamente una forma inequilateral, con el borde anterior más largo y agudo que el posterior y el borde ventral semicircular (Fig. 5A). En ejemplares entre 165 y $200 \mu \mathrm{m}$ LT, el umbo es redondeado y amplio; en ejemplares cercanos a los $220 \mu \mathrm{m}$ LT, el umbo se hace angular y hacia el final del período larval, $300 \mu \mathrm{m}$ LT, se observa prominente. Los hombros son largos, rectos y bajos. La prodisoconcha I se observa homogénea, mientras que la prodisoconcha II exhibe visibles líneas concéntricas de crecimiento. La línea paleal e impresiones de los músculos aductores son notorias ya a los $150 \mu \mathrm{m}$ LT (Fig. 5A). Las valvas, de un tono amarillento entre la línea paleal y el margen ventral, son transparentes y macizas. El provínculo es grueso, presenta 2 dientes cardinales rectangulares: uno anterior en la valva derecha y otro central en la valva izquierda. Estos dientes no exhiben mayores cambios durante el período larval (Fig. 5B y D). La hendidura del ligamento es posterior, poco profunda y poco perceptible (Fig. 5B y D). El sistema lateral de charnela se distingue en todos los ejemplares; está compuesto por una canaleta anterior y un reborde posterior en la valva derecha, y un reborde anterior y una canaleta posterior en la valva izquierda (Fig. 5B y D).

En las postlarvas, el margen de la concha es discontinuo, con forma trapezoidal, muy irregular e inequilateral, con el borde posterior más largo que el anterior. El margen ventral es inclinado hacia la parte posterior y, la mayoría de las veces, es recto (Fig. 5A). El umbo es prominente. Los hombros son rectos, bajos; el 


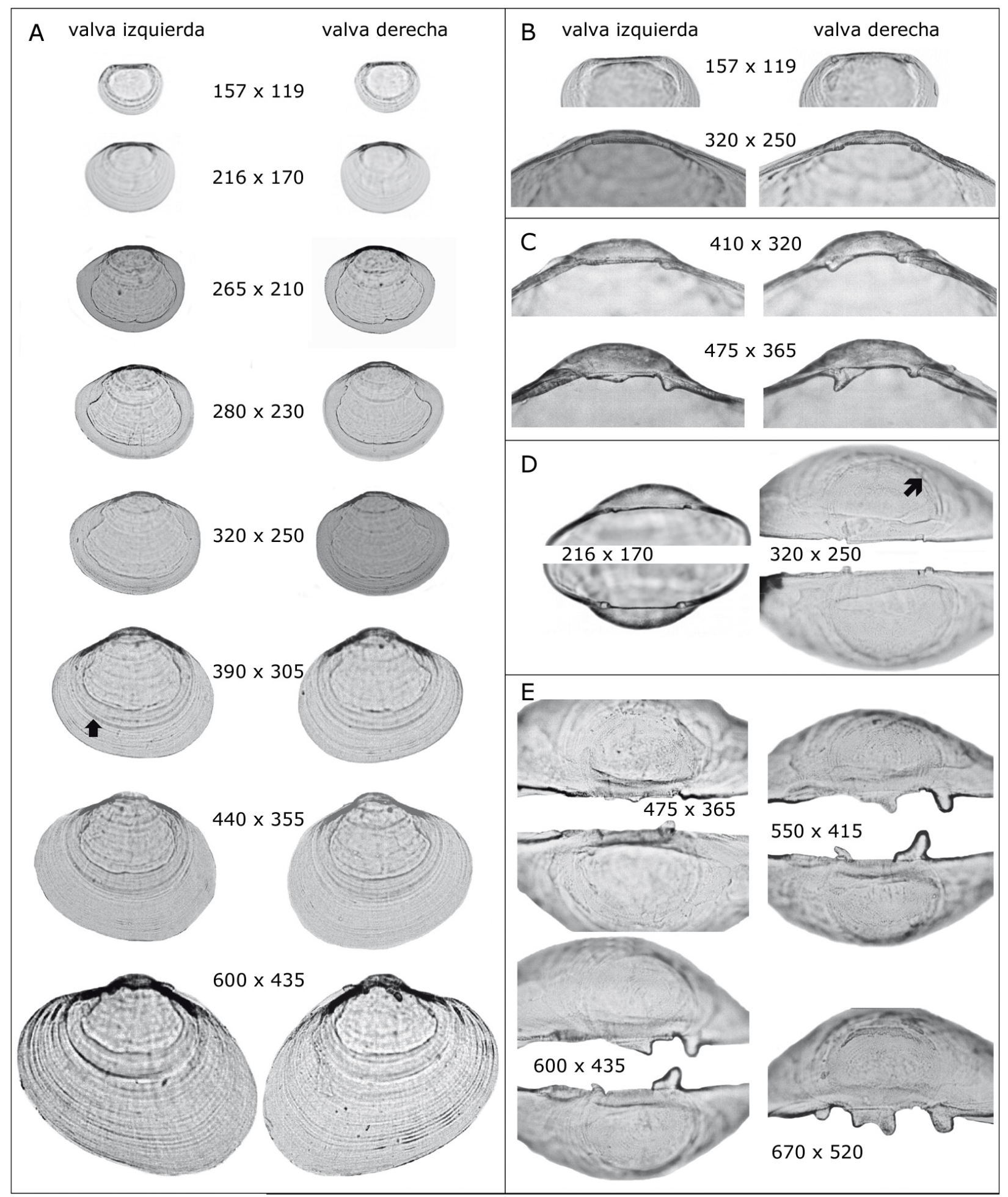

Figura 4. Ensis macha. A. Vista lateral externa de la concha. B y C. Vista lateral interna de la concha larval y postlarval, respectivamente. D y E. Vista dorsal de la concha larval y postlarval, respectivamente. Vd: valva derecha. Vi: valva izquierda. prodisoconcha I - prodisoconcha II. Dimensiones (LT x AT) en $\mu \mathrm{m}$ / Ensis macha. A. External lateral view of the shell. B and C. Internal lateral view of the larval and postlarval shell, respectively. D and E. Dorsal view of the larval and postlarval shell, respectively. Vd: right valve. Vi: left valve. : metamorphic line. $\boldsymbol{\lambda}$ : prodissoconch I-prodissoconch II limit. Dimensions (LT x AT) in $\mu \mathrm{m}$ 


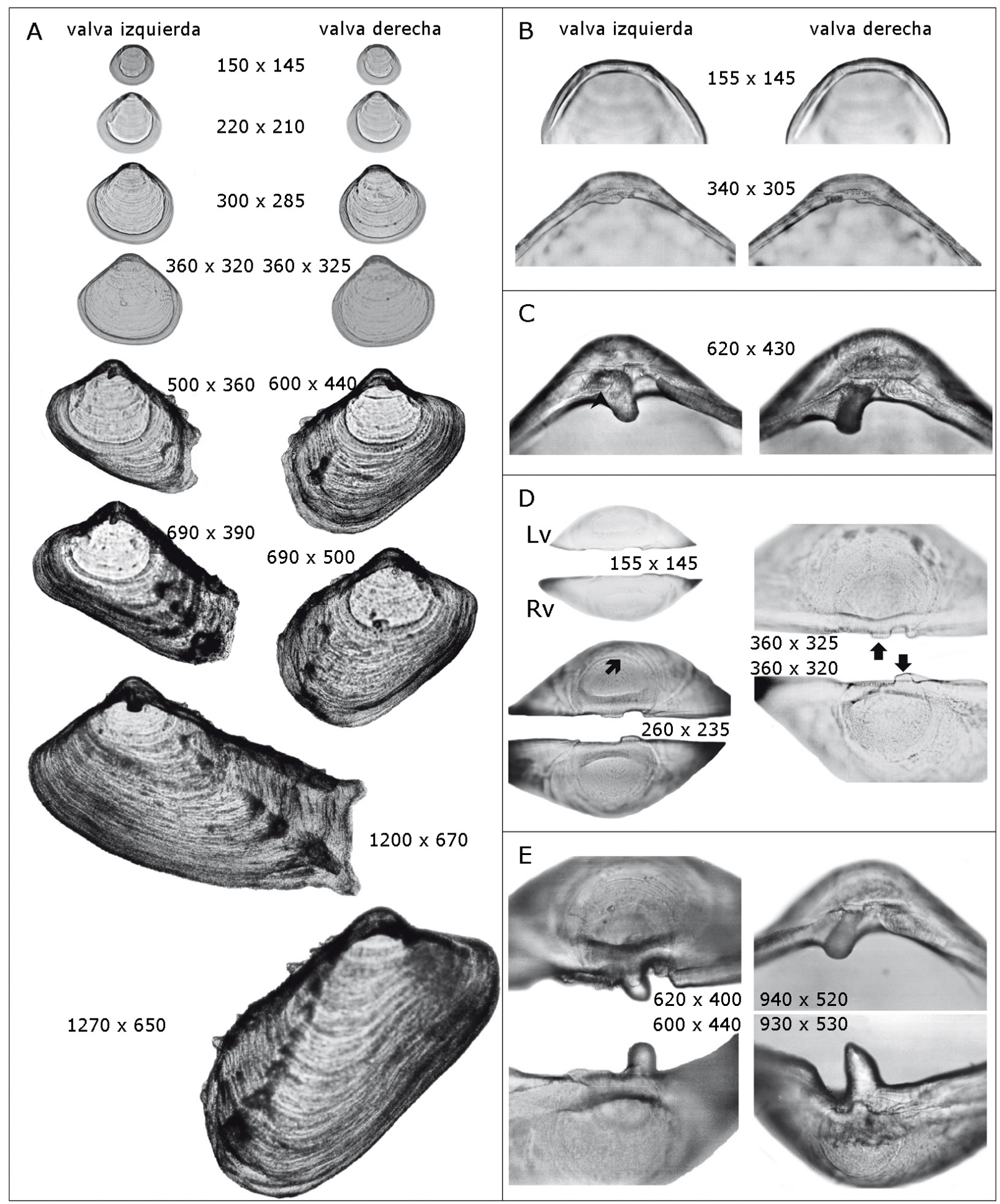

Figura 5. Hiatella solida. A. Vista lateral externa de la concha. B y C. Vista lateral interna de la concha larval y postlarval, respectivamente. D y E. Vista dorsal de la concha larval y postlarval, respectivamente. Vd: valva derecha. Vi: valva izquierda. $\$$ : diente anterior. $\uparrow$ : diente central. $A$ : hendidura del ligamento. $\boldsymbol{\pi}$ : límite prodisoconcha I - prodisoconcha II. Las valvas provienen de diferentes ejemplares. Dimensiones (LT x AT) en $\mu \mathrm{m} /$ Hiatella solida. A. External lateral view of the shell. B and C. Internal lateral view of the larval and postlarval shell, respectively. D and E. dorsal view of the larval and postlarval shell, respectively. Vd: right valve. Vi: left valve. groove. $\boldsymbol{\lambda}$ : prodissoconch I - prodissoconch II limit. Valves are from different specimens. Dimensions (LT x AT) in $\mu \mathrm{m}$ 
hombro posterior es más largo que el anterior (Fig. 5A). La disoconcha presenta líneas concéntricas evidentes y desarrolla 2 cordones radiales con espinas hacia la zona dorso-posterior (Fig. 5A). Las valvas son incoloras, opacas y macizas. El provínculo es grueso. En el estado postlarval, los dientes larvales se alargan: el diente anterior de la valva derecha se extiende hacia la valva opuesta y el diente central de la valva izquierda lo hace hacia la parte anterior (Fig. 5C y E). Alrededor de los 1200 $\mu \mathrm{m}$ LT de la concha, el diente cardinal anterior de la valva derecha se observa piriforme. La hendidura del ligamento tiene forma lanceolada, prolongada hacia la parte posterior del provínculo (Fig. 5C y E). El sistema lateral de la charnela no se desarrolla, manteniendo la forma básica descrita para larvas (Fig. 5C y E).

\section{MORFOMETRÍA}

La morfometría de las prodisoconchas I y II y de los hombros de las larvas y postlarvas de Venus antiqua, Mulinia edulis, Ensis macha y Hiatella solida se muestra en la Tabla 1. La relación entre el largo y el alto de las conchas larval y postlarval en todas las especies fue lineal. Los resultados de las relaciones lineales de AT / LT y LHp / LHa larvales y postlarvales se muestran en la Tabla 2. Las formas de la concha larval de las cuatro especies presentaron diferencias significativas entre ellas $(P<0,05 ; \mathrm{F}=194,7 ; \mathrm{n}=429)$.

Las 4 especies estudiadas presentaron diferencias significativas $(P<0,05 ; \mathrm{F}=5,5 ; \mathrm{n}=429)$ en las tasas de cambio de talla en altura con respecto del largo de la concha. Los valores de la pendiente $(\mathrm{m})$ mostraron que

Tabla 1. Morfometría larval y postlarval de Venus antiqua, Mulinia edulis, Ensis macha y Hiatella solida / Larval and postlarval morphometry of Venus antiqua, Mulinia edulis, Ensis macha and Hiatella solida

\begin{tabular}{|c|c|c|c|c|c|c|}
\hline & Estado & Dimensión & Rango $(\mu \mathrm{m})$ & $\begin{array}{l}\text { Prom. } \\
(\mu \mathrm{m})\end{array}$ & $\begin{array}{l}\text { d.e. } \\
(\mu \mathrm{m})\end{array}$ & $\mathrm{n}$ \\
\hline \multirow{12}{*}{ 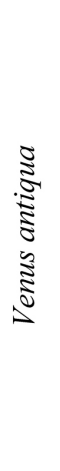 } & \multirow[t]{7}{*}{$\mathrm{L}$} & LT & $123-300$ & 268 & 24 & 127 \\
\hline & & AT & $92-280$ & 244 & 25 & 127 \\
\hline & & LHa & $93-175$ & 114 & 16 & 10 \\
\hline & & LHp & $80-150$ & 104 & 17 & 12 \\
\hline & & LPI & $95-125$ & 112 & 7 & 132 \\
\hline & & LPII & $275-315$ & 298 & 14 & 9 \\
\hline & & LPV & $75-95$ & 79 & 6 & 12 \\
\hline & \multirow[t]{5}{*}{$\mathrm{P}$} & LT & $330-370$ & 348 & 17 & 9 \\
\hline & & $\mathrm{AT}$ & $295-330$ & 314 & 14 & 9 \\
\hline & & LHa & $100-175$ & 128 & 21 & 9 \\
\hline & & LHp & $87-150$ & 115 & 20 & 9 \\
\hline & & LPv & $75-95$ & 82 & 8 & 9 \\
\hline \multirow{12}{*}{ 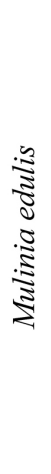 } & \multirow[t]{7}{*}{$\mathrm{L}$} & LT & $140-325$ & 213 & 54 & 112 \\
\hline & & $\mathrm{AT}$ & $120-320$ & 193 & 51 & 113 \\
\hline & & LHa & $42-127$ & 86 & 23 & 105 \\
\hline & & LHp & $37-115$ & 70 & 19 & 105 \\
\hline & & LPI & $80-95$ & 86 & 4 & 155 \\
\hline & & LPII & $265-325$ & 282 & 18 & 42 \\
\hline & & $\mathrm{LPV}$ & $42-62$ & 54 & 5 & 105 \\
\hline & \multirow[t]{5}{*}{$\mathrm{P}$} & LT & $300-580$ & 406 & 66 & 42 \\
\hline & & AT & $270-380$ & 348 & 55 & 41 \\
\hline & & LHa & $100-187$ & 132 & 23 & 35 \\
\hline & & LHp & 77-175 & 121 & 21 & 35 \\
\hline & & LPv & $55-75$ & 66 & 6 & 35 \\
\hline
\end{tabular}

\begin{tabular}{|c|c|c|c|c|c|c|}
\hline & Estado & Dimensión & Rango $(\mu \mathrm{m})$ & $\begin{array}{l}\text { Prom. } \\
(\mu \mathrm{m})\end{array}$ & $\begin{array}{l}\text { d.e. } \\
(\mu \mathrm{m})\end{array}$ & $\mathrm{n}$ \\
\hline \multirow{12}{*}{ 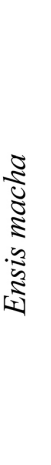 } & \multirow[t]{7}{*}{$\mathrm{L}$} & LT & $157-325$ & 280 & 32 & 45 \\
\hline & & AT & $119-255$ & 225 & 27 & 45 \\
\hline & & LHa & $75-100$ & 94 & 10 & 7 \\
\hline & & LHp & $62-87$ & 75 & 7 & 7 \\
\hline & & LPI & $100-125$ & 112 & 5 & 99 \\
\hline & & LPII & $250-335$ & 310 & 17 & 60 \\
\hline & & $\mathrm{LPv}$ & $72-80$ & 75 & 2 & 7 \\
\hline & \multirow[t]{5}{*}{$\mathrm{P}$} & $\mathrm{LT}$ & $280-670$ & 439 & 78 & 62 \\
\hline & & $\mathrm{AT}$ & $225-520$ & 339 & 55 & 62 \\
\hline & & LHa & $92-217$ & 134 & 24 & 51 \\
\hline & & LHp & $67-192$ & 107 & 23 & 51 \\
\hline & & $\mathrm{LPv}$ & $75-100$ & 84 & 5 & 51 \\
\hline \multirow{12}{*}{ 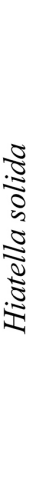 } & \multirow[t]{7}{*}{ L } & LT & $145-370$ & 256 & 62 & 144 \\
\hline & & $\mathrm{AT}$ & $125-345$ & 236 & 56 & 144 \\
\hline & & LHa & $75-200$ & 139 & 37 & 115 \\
\hline & & LHp & $62-187$ & 128 & 35 & 115 \\
\hline & & LPI & $65-95$ & 79 & 5 & 197 \\
\hline & & LPII & $285-380$ & 336 & 23 & 55 \\
\hline & & $\mathrm{LPv}$ & $50-75$ & 64 & 5 & 116 \\
\hline & \multirow[t]{5}{*}{$\mathrm{P}$} & $\mathrm{LT}$ & $440-1270$ & 708 & 195 & 48 \\
\hline & & $\mathrm{AT}$ & $340-670$ & 437 & 78 & 55 \\
\hline & & LHa & $175-375$ & 248 & 46 & 55 \\
\hline & & LHp & $300-1050$ & 509 & 159 & 51 \\
\hline & & LPv & $55-75$ & 64 & 4 & 55 \\
\hline
\end{tabular}

$\mathrm{L}=$ larval; $\mathrm{P}=$ postlarval; $\mathrm{LT}=$ largo total; $\mathrm{AT}=$ alto total; $\mathrm{LHa}=$ largo hombro anterior; $\mathrm{LHp}=$ largo hombro posterior; $\mathrm{LPI}=$ Largo prodisoconcha I; LPII= Largo prodisoconcha II; LPv= largo provínculo; Prom.= promedio; d.e.= desviación estándar; $\mathrm{n}=$ número de individuos medidos 
larvas y postlarvas de E. macha $(\mathrm{m}=0,82$ y $\mathrm{m}=0,69$, respectivamente) y $H$. solida $(\mathrm{m}=0,90$ y $\mathrm{m}=0,39$, respectivamente) tienen crecimiento alométrico, en cambio, en larvas de V. antiqua y $M$. edulis, el crecimiento es casi isométrico $(\mathrm{m}=0,99 \mathrm{y} \mathrm{m}=0,90$, respectivamente). Comparativamente, E. macha presentó la menor tasa de cambio de talla en estado larval.

Las postlarvas de M. edulis, E. macha y H. solida, en este estado, son significativamente diferentes en forma $(P<0,05 ; \mathrm{F}=20,5 ; \mathrm{n}=151)$ al comparar el incremento del alto de la concha para un mismo largo. Las 4 especies presentaron diferencias significativas $(P<0,05 ; \mathrm{F}=70,8$; $\mathrm{n}=151)$ en las tasas de cambio de talla en altura con respecto del largo de la concha postlarval. Los valores de la pendiente $(\mathrm{m})$ evidenciaron un crecimiento alométrico durante este estado. La mayor y menor tasa de crecimiento postlarval fue de 0,79 y 0,39 , en M. edulis y H. solida, respectivamente.

Tabla 2. Relaciones lineales de larvas y postlarvas de Venus antiqua, Mulinia edulis, Ensis macha y Hiatella solida entre AT/ LT y LHp/

LHa / Larval and postlarval lineal regressions of Venus antiqua, Mulinia edulis, Ensis macha and Hiatella solida between AT/LT and $\mathrm{LHp} / \mathrm{LHa}$

\begin{tabular}{|c|c|c|c|c|c|}
\hline \multicolumn{2}{|c|}{ Estado } & \multicolumn{2}{|c|}{$\begin{array}{c}\mathrm{AT} / \mathrm{LT} \\
\left(\mathrm{r}^{2} ; \mathrm{n}\right)\end{array}$} & \multicolumn{2}{|c|}{$\begin{array}{l}\mathrm{LHp} / \mathrm{LHa} \\
\quad\left(\mathrm{r}^{2} ; \mathrm{n}\right)\end{array}$} \\
\hline$\stackrel{2}{3}$ & $\mathrm{~L}$ & $\begin{array}{l}\mathrm{AT}=0,99 \\
(0,88 ; 127)\end{array}$ & LT-22,07 & $\begin{array}{l}\mathrm{LHp}=0,98 \\
(0,90 ; 10)\end{array}$ & LHa-7,51 \\
\hline ป & $\mathrm{P}$ & $\begin{array}{l}\mathrm{AT}=0,74 \\
(0,80 ; 9)\end{array}$ & $\mathrm{LT}+55,11$ & $\begin{array}{l}\mathrm{LHp}=0,83 \\
(0,83 ; 9)\end{array}$ & $\mathrm{LHa}+9,20$ \\
\hline$\stackrel{2}{\frac{8}{3}}$ & $\mathrm{~L}$ & $\begin{array}{l}\mathrm{AT}=0,90 \\
(0,99 ; 113)\end{array}$ & LT-1,65 & $\begin{array}{l}\mathrm{LHp}=0,75 \\
(0,84 ; 105)\end{array}$ & $\mathrm{LHa}+4,73$ \\
\hline 离 & $\mathrm{P}$ & $\begin{array}{l}\mathrm{AT}=0,79 \\
(0,93 ; 41)\end{array}$ & $\mathrm{LT}+24,35$ & $\begin{array}{l}\mathrm{LHp}=0,65 \\
(0,51 ; 35)\end{array}$ & $\mathrm{LHa}+34,90$ \\
\hline$\frac{0}{3}$ & $\mathrm{~L}$ & $\begin{array}{l}\mathrm{AT}=0,82 \\
(0,94 ; 45)\end{array}$ & LT-3,48 & $\begin{array}{l}\mathrm{LHp}=0,25 \\
(0,11 ; 7)\end{array}$ & $\mathrm{LHa}+51,53$ \\
\hline 恣 & $\mathrm{P}$ & $\begin{array}{l}\mathrm{AT}=0,69 \\
(0,97 ; 62)\end{array}$ & $\mathrm{LT}+36,58$ & $\begin{array}{l}\mathrm{LHp}=0,75 \\
(0,61 ; 51)\end{array}$ & $\mathrm{LHa}+6,44$ \\
\hline$\frac{8}{8}$ & $\mathrm{~L}$ & $\begin{array}{l}\mathrm{AT}=0,90 \\
(0,98 ; 144)\end{array}$ & $\mathrm{LT}+6,18$ & $\begin{array}{l}\mathrm{LHp}=0,91 \\
(0,95 ; 115)\end{array}$ & $\mathrm{LHa}+1,38$ \\
\hline$\underset{\widetilde{\Xi}}{\tilde{\Xi}}$ & $\mathrm{P}$ & $\begin{array}{l}\mathrm{AT}=0,39 \\
(0,88 ; 48)\end{array}$ & LT-167,61 & $\begin{array}{l}\mathrm{LHp}=2,94 \\
(0,73 ; 51)\end{array}$ & LHa-227,13 \\
\hline
\end{tabular}

$\mathrm{L}=$ larval; $\mathrm{P}=$ postlarval; $\mathrm{AT}=$ alto total $\mathrm{LT}=$ largo total; $\mathrm{LHp}=$ largo hombro posterior; $\mathrm{LHa}=$ largo hombro anterior; $\mathrm{r}^{2}=$ coeficiente de determinación; $\mathrm{n}=$ número de individuos medidos

\section{Discusión}

Los caracteres morfológicos y morfométricos de la concha de los bivalvos permiten, en gran medida, caracterizar las especies y discriminar entre ellas, proporcionando además, de forma indirecta, información acerca del desarrollo, crecimiento, ecología y conducta de los organismos (Ockelmann 1965, Prezant 1990). Aunque estos caracteres han permitido la identificación de larvas y postlarvas en numerosas especies de bivalvos, hay consenso en la gran dificultad para discriminar entre ellas durante el estado de velígera temprana (larva D) ya que una larva de talla inferior a $150 \mu \mathrm{m}$, solo presenta prodisoconcha I, con una charnela recta casi sin desarrollo denticular (Zardus \& Martel 2002). Esto se corrobora en este estudio ya que las conchas más pequeñas de $M$. edulis, E. macha y H. solida presentan charnelas sin dentición definida. Con el desarrollo del umbo, la charnela y la prodisoconcha II, en el estado de velígera umbonada $(>150 \mu \mathrm{m})$, se ve facilitada la asignación de las larvas a distintos niveles taxonómicos. Rees (1950) señala que las larvas pueden ser asignadas a superfamilias por su charnela y a género y especie por la forma de la concha y su textura. En el caso de las especies estudiadas, se esperaría mayor dificultad para discriminar, solo por su forma general, las larvas de $V$. antiqua de las de M. edulis (Fig. 6); sin embargo, la diferencia en la longitud de la prodisoconcha I entre ambas especies y especialmente el aparato charnelar permitirían reconocerlas ya que cada una de estas especies exhibe las características provinculares propias de la familia a la que pertenece (Kasyanov et al. 1998).

\section{VENUS ANTIQUA}

El desarrollo de la concha larval de $V$. antiqua es similar al de otras especies de la familia Veneridae, estudiadas por Jorgensen (1946), Ansell (1962), LaBarbera \& Chanley (1970), Le Pennec \& Lucas (1970), Le Pennec (1978), Moueza et al. (1999, 2006), tanto en su forma general como en las características provinculares (Rees 1950). Sin embargo, se encontrarían diferencias en el desarrollo de la charnela en postlarvas ya que Le Pennec (1978) indica para otra especie de Venus que la dentición larval persistiría hasta los $500 \mu \mathrm{m} \mathrm{LT}$, sin embargo, en V. antiqua, ya a los $300 \mu \mathrm{m}$ LT, se observó el desarrollo de un diente central cubriendo los dientes de la charnela larval.

El largo promedio de la prodisoconcha I de V. antiqua $(112 \mu \mathrm{m})$ es similar al registrado por Olavarría et al. (1996) $(113,5 \mu \mathrm{m})$ en la misma especie, pero es levemente superior 


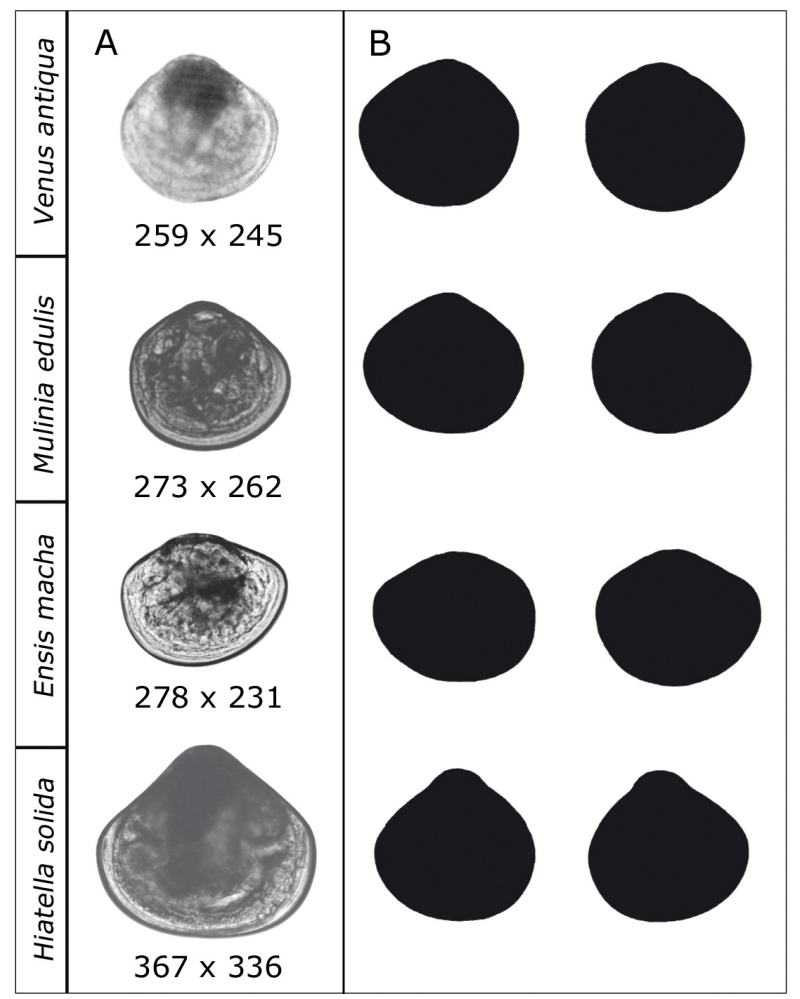

Figura 6. A. Larvas pedivelígeras completas de V. antiqua, M. edulis, E. macha y H. solida. B. Comparación de la forma entre valvas izquierdas y derechas de larvas de aproximadamente $280 \mu \mathrm{m}$ de largo, de cada especie. Dimensiones (LT x AT) en $\mu \mathrm{m} / \mathrm{A}$. Complete pediveliger larvae of $\mathrm{V}$. antiqua, M. edulis, E. macha and $\mathrm{H}$. solida. B. Comparison of shapes between left and right larval valves of approximately $280 \mu \mathrm{m}$ in length of each species. Dimensions (LT $x$ AT) in $\mu \mathrm{m}$

al registrado en otros venéridos (90 a $108 \mu \mathrm{m}$ ) (Jorgensen 1946, Ansell 1962, Le Pennec 1978). Ockelmann (1965) propone que las especies con una talla de prodisoconcha I de 70 a $150 \mu \mathrm{m}$ de largo y un diámetro del ovocito de 45 a $85 \mu \mathrm{m}$ presentarían desarrollo planctotrófico. Según Padilla \& Olivares (1986), el diámetro del ovocito en $V$. antiqua sería de $70 \mu \mathrm{m}$, por lo tanto, sería una especie con desarrollo larval planctotrófico, de duración entre 30 y 35 días (Olavarría 1996). La relación morfométrica lineal entre el alto y el largo total de la concha larval de V. antiqua es similar a la obtenida por Le Pennec \& Lucas (1970) para otras especies de Veneridae. La talla de asentamiento (largo de prodisoconcha II) de V. antiqua ha sido registrada tanto en ejemplares recolectados del medio (Stead 1992) como cultivados (Campos et al. 1993², Olavarría et al. 1996), con un rango de variación entre 200 y 309,8 $\mu \mathrm{m}$ LT, confirmando que esta dimensión es muy variable para ser utilizada como carácter discriminante pero sí permite establecer el rango de talla en el que puede ocurrir la metamorfosis.

\section{MULINIA EDULIS}

Las larvas de esta especie presentan en general las características señaladas para la familia Mactridae, en la cual el margen posterior de la concha se inclina y es casi perpendicular al margen ventral, con la zona de los hombros y el umbo algo triangular y con el hombro anterior más largo y agudo que el posterior (Jorgensen 1946, Sullivan 1948, Rees 1950, Chanley \& Andrews 1971, Kasyanov et al. 1998).

El provínculo de Mulinia edulis es de tipo 'c', según la clasificación de Rees (1950), similar al de Mactra corallina pero diferente al de otros máctridos, que presentarían un provinculo tipo 'a' según este autor.

El rango de talla de la prodisoconcha I de $M$. edulis $(80$ a $95 \mu \mathrm{m}$ ) es similar al registrado por Fuentes (1988) y Farías (1997) y es superior al señalado para M. lateralis por Chanley \& Andrews (1971). De acuerdo con la relación propuesta por Ockelmann (1965), M. edulis tendría un desarrollo larval planctotrófico, por la talla de su prodisoconcha I y porque el diámetro de los ovocitos variaría entre 50 y $59 \mu \mathrm{m}$ (Fuentes 1988, Gutiérrez et al. $2001)^{3}$ y tendría una duración larval entre 14 y 17 días (Farías 1997, Oliva et al. 2013).

Mulinia edulis tiene una talla de metamorfosis en el rango reportado por Fuentes (1988) y Reimers (2003) para la misma especie, pero es mayor a la de M. lateralis (185$240 \mu \mathrm{m})$ (Chanley \& Andrews 1971).

${ }^{2}$ Campos B, R Guerra \& G Bellolio. 1993. Bases biotecnológicas para la obtención masiva de juveniles de almejas de importancia económica. Informe Técnico FONDECYT 91-502: 1-28.

${ }^{3}$ Gutiérrez R, A Célis \& B Campos. 2001. Efectos del tamaño de ovocitos en la viabilidad de larvas de la almeja taquilla Mulinia edulis. XXI Congreso de Ciencias del Mar, Viña del Mar (resúmenes), p. 136. 


\section{ENSIS MACHA}

Esta especie se asigna actualmente a la familia Pharidae (Da Costa et al. 2008, 2010); sin embargo, sus larvas y postlarvas han sido estudiadas como pertenecientes a la familia Solenidae (Lebour 1938, Jorgensen 1946, Sullivan 1948, Rees 1950 y Chanley \& Andrews 1971) o Cultellidae (Kasyanov et al. 1998).

La morfología de la concha larval de E. macha es semejante a la de otras especies de Ensis (Da Costa et al. 2010), sin embargo difiere en las demarcaciones de la línea paleal y de la prodisoconcha II (Chanley \& Andrews 1971). De acuerdo con Rees (1950), el umbo en esta familia se caracteriza por ser más alto en la valva derecha, lo que se corrobora en E. macha (Fig. 4). La charnela larval de $E$. macha es de tipo 'b' y 'd' de Rees (1950), en la valva derecha e izquierda, respectivamente y coincidiría con la de E. directus (Chanley \& Andrews 1971). La charnela tipo 'b' se caracteriza por presentar un diente rectangular y la de tipo 'd' por presentar dientes tuberculares que encajan en hendiduras de la valva contraria. Las postlarvas de $E$. macha presentan una charnela similar a la de $E$. directus (Rees 1950, Sullivan 1948). Rees (1950) estableció que en el género Ensis el ligamento comenzaría a desarrollarse alrededor de los $280 \mu \mathrm{m}$ LT. En E. macha los primeros vestigios de ligamento se observaron alrededor de los $335 \mu \mathrm{m}$ LT, pero es probable que éste se desarrolle a un tamaño inferior y no se haya observado debido a su desintegración durante la limpieza de las valvas.

Rees (1950) no describe dientes laterales para la superfamilia Solenacea, sin embargo, en uno de sus esquemas de charnela se pueden observar 2 proyecciones laterales, una a cada lado de la valva izquierda en un ejemplar de $400 \mu \mathrm{m} \mathrm{LT}$; en E. macha, el desarrollo de estas proyecciones es más tardío y comienza en la valva izquierda, alrededor de los $475 \mu \mathrm{m}$ LT.

El rango de talla de metamorfosis de E. macha (250$335 \mu \mathrm{m})$ coincide con el señalado por Lépez (2006) y se encuentra en el rango descrito para otras especies del género: $220 \mu \mathrm{m}$ en $E$. directus (Chanley \& Andrews 1971), 325 a $382 \mu \mathrm{m}$ en E. arcuatus (Da Costa 2008) y 301-362 $\mu \mathrm{m}$ en E. siliqua (Da Costa et al. 2010).

Al relacionar la longitud de la prodisoconcha I (100$125 \mu \mathrm{m})$, con el diámetro del ovocito, reportado por Avellanal et al. (2002) $(50-51 \mu \mathrm{m})$, se puede concluir que E. macha presentaría desarrollo planctotrófico, con una duración de su período larval entre 17 y 24 días (Olave et al. 19994, Lépez 2006), similar a la de E. arcuatus (20 días) (Da Costa et al. 2011) y algo superior a la de E. siliqua (14-15 días) (Da Costa et al. 2010), sin considerar la temperatura a la que ocurren los respectivos desarrollos.

\section{HIATELLA SOLIDA}

Las larvas y postlarvas de la familia Hiatellidae, a la cual es actualmente asignada Hiatella solida, fueron anteriormente estudiadas como pertenecientes a la familia Saxicavidae (Lebour 1938, Jorgensen 1946, Sullivan 1948, Rees 1950).

La talla de aparición del umbo en $H$. solida coincidiría con el rango del alto $(130-210 \mu \mathrm{m})$ propuesto para $H$. arctica por Sullivan (1948). Según Savage \& Goldberg (1976), en Hiatella sp., el umbo estaría presente ya en larvas de $115 \mu \mathrm{m}$ LT, sin embargo este dato no se pudo comparar ya que en nuestro estudio no se contó con ejemplares de ese tamaño. El umbo de las postlarvas de $H$. solida solo tendría semejanza con el de $H$. (= Saxicava) arctica, aunque en esta última especie, el margen del umbo mantiene una línea continua con el margen valvar, lo que no ocurre en $H$. solida, en la cual el umbo es claramente prominente. En las larvas de H. solida, los hombros se presentan rectos hasta los $200 \mu \mathrm{m}$ LT, mientras que en $H$. (Saxicava) rugosa, el hombro posterior se observa más redondeado que el anterior. Las únicas especies que se asemejarían en forma de hombros larvales a $H$. solida serían $H$. arctica y Hiatella sp., cuando presentan tallas entre 200 y $300 \mu \mathrm{m}$ LT (Sullivan 1948, Savage \& Goldberg 1976),

Las postlarvas del género Hiatella se caracterizan por un desproporcionado elongamiento del hombro posterior (Lebour 1938, Jorgensen 1946, Hayami \& Kase 1993, Flyachinskaya \& Lezin 2008, Schejter et al. 2010) lo que se confirma en $H$. solida. Sin embargo, se registraron grados de inclinación diferentes en los hombros entre $H$. solida de Putemún y $H$. solida de Valparaíso. Estas diferencias morfológicas son esperables en individuos de distintas localidades, debido a las condiciones ambientales imperantes (Márquez \& Van der Molen 2011).

La charnela larval de los ejemplares de Hiatella estudiados aquí, coincide con el tipo 'b' de Rees (1950), con un diente rectangular anterior derecho y un diente rectangular central izquierdo. Este tipo de charnela estaría

${ }^{4}$ Olave S, P Cárcamo, M Díaz \& E Pacheco. 1999. Cultivo larvario y postlarvario del huepo (Ensis macha). XIX Congreso de Ciencias del Mar, Antofagasta, resúmenes, p. 155. 
presente al menos desde los $165 \mu \mathrm{m}$ de largo total, talla que no coincidiría con los $300 \mathrm{~mm}$ propuestos para $H$. (Saxicava) rugosa por Jorgensen (1946) como la talla de diferenciación del provínculo. El sistema lateral de charnela de $H$. solida es similar al descrito para otras especies del género (Rees 1950, Flyachinskaya \& Lezin 2008, Schejter et al. 2010). El característico color amarillento alrededor del borde de la concha larval en esta especie es similar a lo descrito para $H$. arctica (Rees 1950), sin embargo, la prodisoconcha de una misma especie puede presentar distintas coloraciones lo que indica que esta característica no es decisiva al momento de clasificar taxonómicamente a los ejemplares de este género.

La disoconcha de $H$. solida se asemeja a la de $H$. arctica, $H$. orientalis y $H$. meridionalis, al presentar todas ellas un desarrollo de espinas posteriores (Hayami \& Kase 1993, Flyachinskaya \& Lezin 2008), lo que permite una fácil discriminación con otras postlarvas de bivalvos presentes en muestras bentónicas.

Savage \& Goldberg (1976) son los únicos investigadores que han estudiado la relación entre largo y alto en Hiatella sp., sin embargo sus resultados no se pueden comparar con los nuestros, pues los rangos de talla analizados (86$96 \mathrm{~mm}$ LT) fueron diferentes al de este trabajo (145-370 mm LT).

La talla de la prodisoconcha I y la talla de metamorfosis (prodisoconcha II) de $H$. solida son similares a las de otras especies del género (Jorgensen 1946, Rees 1950, Hayami \& Kase 1993, Flyachinskaya \& Lezin 2008) pero es inferior a la de H. meridionalis (391,7-486,8 $\mu \mathrm{m}$ LT) (Schejter et al. 2010). Al considerar la relación entre el diámetro del ovocito en el género Hiatella $(50 \mu \mathrm{m})$ (Lebour 1938, Jorgensen 1946, Le Pennec 1978) con la talla de prodisoconcha I de $H$. solida se puede concluir que $H$. solida presenta desarrollo planctotrófico, al igual que $H$. meridionalis (Schetjer et al. 2010).

Si bien para Chile continental se han descrito 2 especies de Hiatella (H. solida y H. antarctica) (Valdovinos 1999), se decidió aunar ambos grupos estudiados bajo el nombre de $H$. solida ya que Dell (1964) propone a $H$. antarctica como sinónimo de $H$. solida y establece que si se encontraran diferencias en la estructura provincular de ejemplares postlarvales, se podrían clasificar como especies distintas, sin embargo en el presente estudio, no se encontraron diferencias en la estructura provincular en los ejemplares examinados. Las nuevas técnicas moleculares de identificación podrían dilucidar si se trata de una o dos especies.
Es probable que al menos las larvas de $V$. antiqua, $M$. edulis y E. macha se encuentren al mismo tiempo en el plancton debido a que sus épocas reproductivas son similares (Lozada \& Bustos 1984, Avellanal et al. 2002). De $H$. solida no hay información publicada acerca de la presencia de larvas en el plancton ni de su época reproductiva; sin embargo, Campos (datos no publicados) ha recolectado larvas en la costa de Chile central, durante la mayor parte del año. En caso de existir coincidencia de todas estas especies en muestras planctónicas, se esperaría un mayor grado de dificultad en diferenciar las larvas de $V$. antiqua con las de M. edulis; para discriminar entre ellas es necesario analizar los hombros y especialmente, el aparato charnelar ya que cada una de las especies estudiadas presenta las características charnelares de la familia a la que pertenece. Otra dificultad en el reconocimiento de las larvas se presenta al coincidir en las muestras de plancton, larvas de géneros y especies diferentes pertenecientes a la misma familia, como puede suceder con la familia Veneridae, la cual tiene varios representantes en aguas de la costa chilena (Valdovinos 1999), todos ellos con estados del desarrollo que no han sido suficientemente descritos con fines de identificación. Según Shanks (2001), una de las mayores limitaciones para lograr la identificación de los estados larvales y postlarvales de bivalvos es la falta de análisis del aparato charnelar de los ejemplares, probablemente porque requiere entrenamiento técnico y largo tiempo de análisis, con el consecuente costo involucrado. Actualmente y de manera complementaria a la identificación morfológica, se están desarrollando y utilizando técnicas moleculares para la identificación (e.g., Martel et al. 2000), sin embargo, dichas técnicas aún requieren su estandarización para que puedan ser aplicadas de manera sistemática en ejemplares capturados del medio. Por lo tanto, seguir avanzando en el conocimiento morfológico de los primeros estadios del desarrollo, tanto planctónicos como bentónicos de moluscos, sigue siendo un desafío para los estudios ecológicos y de biodiversidad que involucren estos estadios.

Todas las especies analizadas son significativamente diferentes en su morfometría y tasa de cambio de talla. Larvas y postlarvas presentaron un crecimiento alométrico, sin embargo, se observaron mayores pendientes en larvas que en postlarvas, indicando un crecimiento más rápido durante el estado larval. Estos resultados concuerdan con lo reportado por Gribben \& Hay (2003), en Panopea zelandica (Hiatellidae), quienes encontraron una relación lineal entre el largo y el alto de 
la concha durante el desarrollo temprano de la especie; por su parte, Hendriks et al. (2005) compararon las pendientes entre el largo y el alto de la concha larval en 9 especies de bivalvos, encontrando también diferencias entre ellas pero con superposición de las líneas de regresión en las especies analizadas, motivo por el cual estos autores señalan que la comparación de las pendientes entre el largo y el alto de la concha no constituiría una técnica adecuada para una concluyente identificación específica. Es posible que los diagramas tridimensionales de la relación largo-ancho-alto de la concha, propuestos por De Schweinitz \& Lutz (1976) entreguen información morfométrica más adecuada para caracterizar las larvas y postlarvas de bivalvos, a nivel específico así como también podrían aportar las técnicas de morfometría geométrica (Ponton et al. 2013).

\section{Agradecimientos}

A Irene Lépez, Ernesto Olavarría y Carolina Jiménez, por la donación de larvas y postlarvas cultivadas; a Leoncio González, por la separación de ejemplares desde muestras de plancton y bentos; a Mauricio Landaeta, por su ayuda con los análisis estadísticos y a tres evaluadores anónimos cuyos valiosos comentarios permitieron mejorar este trabajo.

\section{LITERATURA CITADA}

Ansell D. 1962. The functional morphology of the larval, and the post-larval development of Venus striatula (Da Costa). Journal of the Marine Biological Association of the United Kingdom 42: 419-443.

Avellanal M, E Jaramillo, E Clasing, P Quijón \& H Contreras. 2002. Reproductive cycle of the bivalves Ensis macha (Molina, 1782) (Solenidae), Tagelus dombeii (Lamarck, 1818) (Solecurtidae), and Mulinia edulis (King, 1831) (Mactridae) in Southern Chile. The Veliger 45(1): 33-44.

Bellolio G, K Lohrmann \& E Dupré. 1993. Larval morphology of the scallop Argopecten purpuratus as revealed by scanning electron microscopy. The Veliger 36(4): 332-342.

Bellolio G, P Toledo \& B Campos. 1994. Morfología de la concha larval y postlarval del ostión Argopecten purpuratus (Lamarck, 1819) (Bivalvia, Pectinidae) en Chile. Revista Chilena de Historia Natural 67(2): 229-237.

Bownes S, NP Barker \& CD McQuaid. 2008. Morphological identification of primary settlers and post-larvae of three mussel species from the coast of South Africa. African Journal of Marine Science 30(2): 233-240.

Brink L. 2001. Mollusca: Bivalvia. In: Shanks A (ed). An identification guide to the larval marine invertebrates of the Pacific Northwest, pp. 131-149. Oregon State University Press, Corvallis.
Campos B \& L Ramorino. 1981. Huevo, larvas y postlarva de Entodesma cuneata (Gray. 1828) (Bivalvia: Pandoracea: Lyonsiidae). Revista de Biología Marina 17(2): 229-251.

Campos B \& L Ramorino. 1990. Larvas y postlarvas de Pholadacea de Chile (Mollusca: Bivalvia). Revista de Biología Marina 25(1): 15-63.

Chanley P \& D Andrews. 1971. Aids for identification of bivalve larvae of Virginia. Malacologia 11(1): 45-119.

Chanley P \& P Dinamani. 1980. Comparative descriptions of some oyster larvae from New Zealand and Chile, and a description of a new genus of oyster, Tiostrea. New Zealand Journal of Marine \& Freshwater Research 14(2): 103-120.

Da Costa F, S Darriba \& D Martínez-Patiño. 2008 Embryonic and larval development of Ensis arcuatus (Jeffreys, 1865) (Bivalvia: Pharidae). Journal of Molluscan Studies 74: 103-109.

Da Costa F, D Martínez-Patiño, J Ojea \& S Nóvoa. 2010. Larval rearing and spat production of the razor clam Ensis siliqua (Bivalvia: Pharidae). Journal of Shellfish Research 29: 347-351.

Da Costa F, S Darriba \& D Martínez-Patiño \& A Guerra. 2011. Culture possibilities of the razor clam Ensis arcuatus (Pharidae: Bivalvia). Aquaculture Research 42: 1549-1557.

Dell RK. 1964. Antarctic and sub-Antarctic Mollusca: Amphineura, Scaphopoda and Bivalvia. Discovery Reports 23: 93-250.

De Schweinitz EH \& RA Lutz. 1976. Larval development of the northern horse mussel, Modiolus modiolus (L.), including a comparison with the larvae of Mytilus edulis L. as an aid in planktonic identification. The Biological Bulletin 150: 348-360.

Farías C. 1997. Efecto de la temperatura y concentración de alimento sobre el crecimiento larval de Mulinia edulis (Bivalvia: Mactridae). Tesis de Biología Marina, Instituto de Oceanología, Universidad de Valparaíso, Valparaíso, 88 pp.

Flyachinskaya LP \& PA Lezin. 2008. Larval and juvenile shell development in the White Sea bivalve Hiatella arctica (Linnaeus, 1767). Invertebrate Zoology 5(1): 39-46. [In Russian, abstract in English].

Fuentes I. 1988. Desarrollo y morfología externa comparada de larvas y postlarvas de Mesodesma donacium y Mulinia sp. (Bivalvia: Mactracea) cultivadas en laboratorio. Tesis de Licenciatura en Ciencias del Mar, Facultad de Ciencias del Mar, Universidad Católica del Norte, Coquimbo, 43 pp.

Goffredi SK, WJ Jones, CA Scholin, R Marin \& RC Vrijenhoek. 2006. Molecular detection of marine invertebrate larvae. Marine Biotechnology 8: 149-160.

Goodsell JG, SC Fuller, AG Eversole, M Castagna \& RA Lutz. 1992. Larval and early postlarval shell morphology of several venerid clams. Journal of the Marine Biological Association of the United Kingdom 72(1): 231-255. 
Gribben PE \& BE Hay. 2003. Larval development of the New Zealand geoduck Panopea zelandica (Bivalvia: Hiatellidae). New Zealand Journal of Marine and Freshwater Research 37(2): 231-239.

Hammer Ø, DAT Harper \& PD Ryan. 2001. PAST: Paleontological statistics software package for education and data analysis. Palaeontologia Electronica 4(1), 9 pp. <http://palaeo-electronica.org/2001_1/past/issue1_01.htm>

Hayami I \& T Kase 1993. Submarine cave Bivalvia from the Ryukyu Islands: systematics and evolutionary significance. The University of Tokyo Bulletin 35: 1-133.

Hendriks IE, LA van Duren \& PMJ Herman. 2005. Image analysis techniques: a tool for the identification of bivalve larvae? Journal of Sea Research 54: 151-162.

Jablonski D \& R Lutz. 1980. Molluscan larval shell morphology. Ecological and paleontological applications. In: Rhoads D \& R Lutz (eds). Skeletal growth of aquatic organisms: biological records of environmental change, $\mathrm{pp}$. 323-377. Plenum Publishing Corporation, New York.

Jorgensen CB. 1946. Lamellibranchia. In: Thorson G (ed). Reproduction and larval development of Danish marine bottom invertebrates with special reference to the planktonic larvae in the Sound (Oresund). Meddelelser fra Kommissionen for Danmarks Fiskeri-og Havundersogelser, Copenhagen, Serie Plankton 4(1): 277-311.

Kasyanov VL, GA Kryuchkova, VA Kulikova \& LA Medvedeva. 1998. Larvae of marine bivalves and echinoderms, 288 pp. Oxonian Press, New Delhi.

LaBarbera M \& P Chanley. 1970. Larval development of Chione cancellata Linne (Veneridae, Bivalvia). Chesapeake Science 11(1): 42-49.

Lebour MV. 1938. Notes on the breeding of some lamellibranchs from Plymouth and their larvae. Journal of the Marine Biological Association of the United Kingdom 23: 119144.

Lépez I. 2006. El cultivo de la navaja chilena Ensis macha. VIII Foro Recursos Mariños e da Acuicultura das Rias Galegas, Illa de A Toxa (O Grove), 6-7 outubro 2005, pp. 347-349. $<\mathrm{http}: / / \mathrm{ww}$ w.fundacionoesa.es/images/stories/ publicaciones/libros/viii_foro_recursos_marinos.pdf >

Le Pennec M. 1978. Génèse de la coquille larvaire et postlarvaire chez divers bivalves marins. These d' Etat, Université de Bretagne Occidentale, Brest, 229 pp.

Le Pennec M. 1980. The larval and post-larval hinge of some families of bivalve molluscs. Journal of the Marine Biological Association of the United Kingdom 60: 601-617.

Le Pennec M \& A Lucas. 1970. Comparative growth and morphology of some venerid larvae (Bivalvia, Veneridae). Malacological Review 3: 175-183.

Lozada E \& H Bustos. 1984. Madurez sexual y fecundidad de Venus antiqua King \& Broderip 1835 en la Bahía de Ancud Mollusca: Bivalvia: Veneridae). Revista de Biología Marina 20(2): 91-112.
Lutz R, J Goodsell, M Castagna, S Chapman, C Newell, H Hidu, R Mann, D Jablonski, V Kennedy, S Siddall, R Goldberg, H Beattie, C Falmagne \& A Chestnut. 1982. Preliminary observations on the usefulness of hinge structures for identification of bivalve larvae. Journal of Shellfish Research 2(1): 65-70.

Lutz RA. 1985. Identification of larvae and postlarvae: a review of recent advances. American Malacological Bulletin, Special Edition 1: 59-78.

Lutz RA \& D Jablonski. 1978. Classification of bivalve larvae and early post-larvae using scanning electron microscopy. American Zoologist 18: 647.

Márquez F \& S Van der Molen. 2011. Intraspecific shell-shape variation in the razor clam Ensis macha along the Patagonian coast. Journal of Molluscan Studies 77(2): 123-128.

Martel AL, LM Auffrey, CD Robles \& BM Honda. 2000. Identification of settling and early postlarval stages of mussels (Mytilus spp.) from the Pacific coast of North America, using prodissoconch morphology and genomic DNA. Marine Biology 137: 811-818.

McManus GB \& LA Katz. 2009. Molecular and morphological methods for identifying plankton: what makes a successful marriage? Journal of Plankton Research 31: 1119-1129.

Moueza M, O Gros \& L Frenkiel. 1999. Embryonic, larval and postlarval development of the tropical clam, Anomalocardia brasiliana (Bivalvia, Veneridae). Journal of Molluscan Studies 65: 73-88.

Moueza M, O Gros \& L Frenkiel. 2006. Embryonic development and shell diferentiation in Chione cancellata (Bivalvia, Veneridae): an ultrastructural analysis. Invertebrate Biology 125: 21-33.

Ockelmann K. 1965. Developmental types in marine bivalves and their distribution along the Atlantic coast of Europe. In: Cox LR \& JF Peake (eds). Proceedings of the First European Malacological Congress (1962). Conchological Society of Great Britain and Ireland and the Malacological Society of London. London, pp. 25-35.

Olavarría E, A Farías \& I Uriarte. 1996. Morfometría y tasas de crecimiento larvario y postlarvario de los bivalvos Venus antiqua (King \& Broderip, 1835) y Gari solida (Gray, 1828) cultivados en laboratorio. Revista de Biología Marina 31(2): 107-116.

Oliva D, A Abarca, R Gutiérrez, Á Celis, L Herrera \& V Pizarro. 2013. Effect of stocking density and diet on growth and survival of post-larvae of the taquilla clam Mulinia edulis cultivated in sand in a hatchery. Revista de Biología Marina y Oceanografía 48(1): 37-44.

Padilla M \& G Olivares. 1986. Evaluación de la madurez vitelogénica en oocitos extirpados de la almeja Venus antiqua antiqua. Revista de Biología Marina 22(1): 61-74.

Pardo LM, D Ampuero \& D Véliz. 2009. Using morphological and molecular tools to identify megalopae larvae collected in the field: the case of sympatric Cancer crabs. Journal of the Marine Biological Association of the United Kingdom 89(3): 481-490. 
PaugamA, C D'Ollone, JC Cochard, P Garen \& M Le Pennec. 2006. The limits of morphometric features for the identification of black-lip pearl oyster (Pinctada margaritifera) larvae. Journal of Shellfish Research 25: 959-967.

Ponton D, L Carassou, S Raillard \& P Borsa. 2013. Geometric morphometrics as a tool for identifying emperor fish (Lethrinidae) larvae and juveniles. Journal of Fish Biology 83: 14-27.

Prezant RS. 1990. Shell and hinge ontogeny of the Antarctic bivalve Lissarca notocardensis. Journal of the Marine Biological Association of the United Kingdom 70: 841-855.

Ramorino L \& B Campos. 1983. Larvas y postlarvas de Mytilidae de Chile (Mollusca: Bivalvia). Revista de Biología Marina 19(2): 143-192.

Rees CB. 1950. The identification and classification of lamellibranch larvae. Hull Bulletins of Marine Ecology 3(19): 73-104.

Reimers C. 2003. Reclutamiento de Mulinia edulis (King \& Boderip 1832) (Bivalvia: Mactridae) en el intermareal de Bahía Yaldad, Quellón, Chiloé. Tesis de Licenciatura en Biología Marina, Universidad Austral de Chile, Valdivia, 51 pp.

Savage N \& R Goldberg. 1976. Investigation of practical means of distinguishing Mya arenaria and Hiatella sp. larvae in plankton samples. Proceedings of the National Shellfisheries Association 66: 42-53.

Schejter L, C Bremec, D Waloszek \& M Escolar. 2010. Recently settled stages and larval developmental mode of the bivalves Zygochlamys patagonica and Hiatella meridionalis in the Argentine sea. Journal of Shellfish Research 29: 63-67.
Shanks AL. 2001. An identification guide to the larval marine invertebrates of the Pacific Northwest, 3147 pp. Oregon State University Press, Corvallis.

Slater J. 2005. Morphological identification of larval king scallops, Pecten maximus (L.) from natural plankton samples. Journal of Shellfish Research 24: 937-949.

Stead R. 1992. Asentamiento de Venus antiqua (Bivalvia: Veneridae) en el intermareal arenoso de Bahía Yaldad, Chiloé. Tesis de Grado de Licenciatura en Biología Marina, Universidad Austral de Chile, Valdivia, 48 pp.

Sullivan C. 1948. Bivalve larvae of Malpeque Bay, P.E.I. Bulletin, Fisheries Research Board of Canada 77: 1-36.

Tremblay MJ, LD Meade \& GV Hurley. 1987. Identification of planktonic sea scallop larvae (Placopecten magellanicus) (Gmelin). Canadian Journal of Fisheries and Aquatic Science 44: 1361-1366.

Turner RD. 1976. Fixation and preservation of molluscan zooplankton. In: Stedman HF (ed). Zooplankton fixation and preservation. Monographs on Oceanographic Methodology 4: 290-294. The UNESCO Press, Paris.

Valdovinos C. 1999. Biodiversidad de moluscos chilenos. Gayana 63(2): 111-164.

Zar J. 1984. Biostatistical analysis, 718 pp. Prentice Hall, Englewood Cliffs.

Zardus JD \& AL Martel. 2002. Phylum Mollusca: Bivalvia. In: Young C, M Sewell \& M Rice (eds). Atlas of marine invertebrate larvae, pp. 289-325. Academic Press, New York. 